\title{
Current Challenges in Labelling for Generic Medicinal Products: Company Core Data Sheet (CCDS) Development and Maintenance
}

\author{
Marion Mueller ${ }^{1} \mathbb{D} \cdot$ David J. Lewis $^{2,3} \mathbb{D} \cdot$ V. Kishore K. Darisi ${ }^{4} \mathbb{D} \cdot$ Sebastian Horn $^{5} \mathbb{D}$
}

Accepted: 24 October 2020 / Published online: 8 December 2020

(c) The Author(s) 2020

\begin{abstract}
Labelling of pharmaceutical products plays a vital role in the safe and effective use of approved medicinal products. This information may be provided to end-users including patients and/or prescribers, and it needs to be made available in multiple formats including printed forms (patient information leaflets, pack inserts, etc.) or web portals of the product, based on national authority guidelines. The Company Core Data Sheet (CCDS) serves as a key document representing the pharmaceutical company's position on the product and is used as a reference document for national labels. Content from national labels may differ from the CCDS for different reasons including implementation of national authority requirements in the serving market and findings from local markets. In the current article, we discuss the process, challenges and key concepts in creating and maintaining CCDS documents for generic products. We highlight key parameters that are worthy of process improvement in generic products' CCDS updates. In addition, we argue that labelling harmonisation across multiple regions, especially safety section-related information, plays a key role in promoting end-user safety and would help communicate risks. We also strongly believe that the topic is worthy of the International Council for Harmonisation of Technical Requirements for Pharmaceuticals for Human Use (ICH) consideration, and propose that this is the key area that requires standardisation and harmonisation.
\end{abstract}

Marion Mueller

Marion.mueller@novartis.com

1 Manager Safety Risk Management, Global Drug Development, Novartis Pharma GmbH, Holzkirchen, Germany

2 Chief Medical Office and Patient Safety, Global Drug Development, Novartis Pharma GmbH, Wehr, Germany

3 Department of Pharmacy, Pharmacology and Postgraduate Medicine, University of Hertfordshire, Hatfield AL10 9AB, Hertfordshire, UK

4 Safety Labeling Manager CDS\&SD, Global Drug Development, Novartis Healthcare, Hyderabad, India

5 Global Drug Development, Head Patient Safety Generics, Novartis Pharma GmbH, Holzkirchen, Germany

\section{Key Points}

The Company Core Data Sheet (CCDS) serves as a key document representing the pharmaceutical company's position on a generic medicinal product.

Some challenges in the generic labelling processes are highlighted, including common procedures and opinions.

Opportunities for improvement are discussed, including labelling harmonisation and IT solutions.

\section{Background}

Labelling of a pharmaceutical product plays a vital role in the safe and effective use of that product. Such information must be provided to end-users, including consumers and prescribers, for decision making on the usage of the pharmaceutical product, and must be available in multiple formats (online and print format) based on the region [e.g. 
USPI (United States Prescribing information) for prescribers and PIL (patient information leaflet) for consumers].

It is the responsibility of the marketing authorisation holders (MAHs) to manage labelling of documents based on relevant national authority requirements and health authority recommendations in the regulatory market.

The Company Core Data Sheet (CCDS) is a master labelling document maintained at a central level by the MAH to represent the company's position on the product, and from which local/national labels will be prepared.

Pharmaceutical products are classified into originator products and generic products. Originator products are medications that were first authorised worldwide based on clinical efficacy and safety studies. Generic products are composed of the same qualitative and quantitative active substances as a reference molecule and are registered based on bioavailability and bioequivalence studies. Typically, there will be no other additional clinical studies for generic products.

Generic product labels are prepared based, broadly, on content from their reference products and additional information from health authorities is also considered.

\section{Current Approach for Generic CCDS Updates}

In general, CCDS preparation starts when the product's Marketing Authorisation is granted, and is prepared by generic companies based on the reference product, followed by updates, which are usually triggered by sources such as:

- Recommendations from health authorities

- Changes in originator label

- Product updates from internal resources (quantitative and qualitative findings from internal databases)

As soon as signals are identified and classified for a product, they will be further evaluated to judge whether or not the information qualifies for updating the CCDS. The MAH performs a medical evaluation to establish a relationship between the use of the product and the conditions that were noted, and it evaluates other factors that could be attributed to the signal.

\section{Current Challenges in CCDS Update}

Currently, pharmaceutical companies prepare/update their generic product CCDS documents with alignment of originator product labels that are publicly available on Health Authority websites. Generic product companies face difficulty in providing justification for the changes made based on originator labels as they did not own complete information about the product and it is difficult to provide justification for label updates as they did not own complete information.

The process becomes especially difficult when the originator updates the labels with their internal safety and clinical data, which is not publicly available at the time of update.

The complexity of harmonising safety information starts with the evaluation of multiple aspects as described below.

\subsection{Identification of Right Originator Label}

Since originators may market their products in multiple countries, it becomes difficult for generic pharmaceutical companies to consider which national label is available in the public domain to consider for CCDS update. Labels that are publicly available might have been prepared based on national authority recommendations, which may not ideally represent the originator company's position. In these instances, generic pharmaceutical companies use multiple major labels to compare information and to establish the company's position in the CCDS.

\subsection{Lack of Harmonised Label Structure}

Currently, there is no defined harmonised template/structure for CCDS. Major pharmaceutical companies prepare their CCDS templates adapting from major labels like USPI or SmPC (Summary of Product Characteristics) style whereas some pharmaceutical companies create either a hybrid model or novel stand-alone model based on their internal decisions.

Since there are no common guidelines for CCDS, their structure is dependent on the pharmaceutical company's discretion [1]. Consequently, differently structured local labels can serve as a reference for signal evaluation or label updates. Comparing these documents, finding new safetyrelevant information, and transferring this information into a CCDS is time-consuming and marginally supported by comparison applications. Fundamentally, it can be debated whether any of the templates are appropriate to maintain safety information.

\subsection{Differences in Procedures}

The process and conventions for updating the CCDS differ between pharmaceutical companies, as no harmonised guideline/structure needs to be followed and companies also follow their convention when updating the CCDS.

Usually, the effort involved in updating generic CCDS is not comparable with originator product label updates. Significant effort is required to update labels; such updates are dependent on internal data, which can be 'owned' by 
multiple functions across the organisation. For originator label updates, cross-functional teams are required for label updates and the process will be treated as a shared effort; for example, the preclinical safety team provides updates from animal studies and the pharmacokinetic department provides updates on product pharmacokinetic parameters from advanced studies or other resources. The major difference between originator and generic labels are the sources of information that are used to update labels. In general, originator companies have more data than the generic companies, as they have access to more data from preclinical and clinical studies, which provides sound scientific justification for adaptation. Consequently, within a generic label health authority recommendations can be implemented, even though an originator label might have been adapted to internal clinical study results that are not available to the public or competitors. Even if both generic and originator companies plan to implement changes according to health authority recommendations, it is possible that, based on differences in processing the variations, the generic label is available more quickly than the originator label.

As a result, health authorities do not normally accept that the generic MAH copies the safety-relevant sections from the referenced originator product label. The MAH must ensure that the product information is up-to-date based on current scientific knowledge, including assessments and recommendations made public via the European medicines web-portal [2].

The non-availability of decision-making documents creates additional efforts for generic companies for considering the changes made by originator companies and sometimes it is difficult to justify the changes, especially if the changes are done based on the originator company's internal data.

\subsection{Differences in Opinion}

The majority of the information in the labels is adapted from originator labels by generic companies; however, there might be differences in opinion while adapting such information based on the company's internal assessment and based on the availability of background data that were considered for label updates.

\subsection{Conversion of Information between Different Formats}

The structure of the label and the process of updating safety information affects the evaluation of that information.

A comparison of the structure of the EU SmPC and the US PI demonstrates the complexity involved in uniting information derived from two major markets (see Table 1). Some sections directly lead to another, while other sections are not represented in both documents or have separate titles. Therefore, it must be carefully evaluated if the content is covered or if it is expressed differently.

\subsection{Class Effects}

A recent example demonstrates the medical evaluation complexity in the risk of severe cutaneous adverse reactions (SCARs) associated with the use of beta-lactam antibiotics. In September 2018, the Canadian regulatory authority (Health Canada) issued a recommendation, aimed at harmonising all beta-lactam labelling by implementing consistent terms for SCAR in all relevant beta-lactam labels [3]. Although the authorities provide supporting publications, these scientific papers described only the suspicion of an association and did not establish a causal relationship between SCAR and all beta-lactams. This is not an issue for the two countries specified, as MAHs in both are obliged to follow the recommendations. However, implementation in the CCDS should be based on a solid foundation of clinical evidence if the company Core Safety Information is to be credible as the basis for regulatory safety variations. Justification documents prepared by MAHs must be acceptable
Table 1 Comparison of EU Summary of Product Characteristics (SmPC) and US Prescribing Information (USPI)

\begin{tabular}{ll}
\hline Section in EU-SmPC & Section in USPI \\
\hline 4.3. Contraindications & 4. Contraindications \\
4.4. Special warnings and precautions for use & 5. Warnings and precaution \\
4.5. Interactions & 7. Drug interactions \\
4.6. Pregnancy and lactation & 8. Use in specific popula- \\
& tion \\
& 8.1. Pregnancy \\
& 8.2. Lactation \\
4.7. Effects on ability to drive and use machines & 8.3 . Females and males \\
Undesirable effects & reproductive potential \\
Overdose & 5. Warnings and precaution \\
\hline
\end{tabular}


for each respective market where a generics company has approval.

The following is a scenario, where the situation was complex and difficult to manage. The originator products (and their respective labels) for a large number of registered beta-lactams were nearly all taken off the market. Evaluation of in-house investigations including broad literature research for published evidence resulted in a high workload as the medical concept (SCAR) includes about 50 Preferred Terms within the Medical Dictionary for Regulatory Activities (MedDRA) terminology (MedDRA Preferred Terms). The application of this broad medical concept across a large group of active ingredients made it especially clear that from the perspective of the global labelling group there was a rapid and significant increase in workload to maintain the CCDS documents for products containing beta-lactams.

As a result, harmonising the labels for a substance class company-internal is nearly impossible when the reference labels are different, outdated or not applicable. Even though additional internal signal detection could provide evidence for a harmonised update in terms of a company position, it is not guaranteed that this can be implemented on a national level.

\subsection{The Company Position}

The content within a nationally approved label for generic products is generally reliant on two primary sources. First, the company's position on the safety profile of the product, which should be backed by supporting data and would typically be described in a CCDS. Second, there is a dependency on the nationally approved text, which is governed by the health authority, which may differ from the company's description in the CCDS. Such content differences may arise for a variety of reasons, including differing medical practices, ethnic or patient variability, availability of competitor products, or differences in opinion between health authorities and the MAH [4].

Therefore, the CCDS for bioequivalent drugs often differ. Concerns about the safety of generics are not limited to patients but extend to other stakeholders including physicians and pharmacists [5]. Physicians are aware of differences in perception and regulators consider new strategies for harmonising bioequivalent labels [6].

\subsection{Compliance}

The basic goal of continuous label maintenance is to make sure that the product updates will swiftly reach end users for better decision making on product use. However, safety data management and management of reference safety information is a common area of non-compliance for many companies. Health authorities usually focus more closely on this during inspections, as failure or delays in the submission of safety variations can affect patient safety.

It should be kept in mind that the CCDS is a document for use by MAHs that does not require approval by any authority. However, the national labels require approval from the responsible authorities.

In case of non-acceptance (i.e. deficiency letter to the $\mathrm{MAH})$, the national labels must undergo an internal review process often requiring additional investigations and evaluation (e.g. literature review, medical evaluation of line listings, etc.). Depending on the complexity of the authority's comments, this evaluation can be very time-consuming.

In case of acceptance, new information must be included in the Marketing Authorisation (product license) and throughout the production system, resulting in the inclusion of up-to-date printed material in package leaflets and other publishing material.

Altogether, it can take several months before newly detected information finally reaches the patient. There are also technical aspects (e.g. 'just-in-time' manufacturing processes) that are not described in this article but may impact the labelling process.

\section{Opportunities for Improvement}

\subsection{Information Technology}

In the area of generic safety labelling, the use of advanced methods like digital systems is not yet widely established and it is the key area where health authorities or pharmaceutical companies must be focused. The need to establish a comprehensive solution to improve the maintenance of CCDSs was identified by the European Medicines Agency (EMA). Unfortunately, the initiation to develop suitable technology was not successful [EMA's Product Information Management (PIM)] [7].

Hock et al. [8] proposed the use of new technologies to support the maintenance of the CCDS using XML-Authoring. The CCDS is viewed as a data container (or database) that holds the textual content of the label in a highly structured, hierarchical manner and provides the possibility to store information beyond the written text (attributes) related to each module of the hierarchy (e.g. pharmaceutical form, route of administration, core/non-core information). The tracking system includes medical and regulatory assessments for all information collected and collated by the MAH. National labelling documents are derived from the CCDS and reflect the national requirements in terms of structure and wording [8].

The approach of maintaining a virtual CCDS within a database, instead of updating single CCDS documents is a promising concept and might gain support from IT vendors. 
Technically, it would be possible to extract information according to the intended purpose. An IT solution, such as the one proposed, could offer customisation according to the MAH's structure and requirements. The system could enable the automated creation of different text documents (or just in terms of reusable modules) and reduce administrative work globally and locally (e.g. text formatting), whilst increasing document compliance. Finally, the accelerated creation of CCDS updates via a repository for safety information would offer:

- Expedited communication between global and national stakeholders

- Improved approval procedures

- Distributed safety information quicker to patients and healthcare professionals (HCPs).

Depending on the structure and workflows within a company, this approach might also serve as a basis for a "One Product Safety Master File" as described by Furlan [9]. The authors describe overlapping and redundant information in multiple safety documents including Periodic Safety Update Reports (PSURs) and Development Safety Update Reports (DSURs), Risk Management Plans (RMPs) and Signal Detection Reports [9]. A database collecting safety information from various pharmacovigilance departments could also provide approved documents for many other objectives such as the scope of case processing and preparation of educational material.

The major objective would be the elimination of manual processes and to make content re-usable, instead of copying and pasting the content into various formats that consequently leads to redundant work.

It must determine what IT solution would best fit a generic approach. The value of an IT solution would be that it could provide a full package of creation, maintenance, tracking, and approval features, as well as a complete audit trail of changes and derivatives. These approaches could start with CCDS maintenance and even bring information to consumers and prescribers in terms of e-labelling approaches.

However, the benefits in terms of cost effectiveness and time savings need to be evaluated carefully. Consideration must be given to the fact that, although changes can be easily implemented, that data entry into a complex IT system and associated approval steps might impact time expenditure.

\subsection{Harmonisation of Labelling}

The creation of a common template and a detailed guideline would be beneficial for the MAHs when creating/updating respective documents but also for the health authority inspectors.
Not all health authorities have the resources to review changes to the national label of each licensed product based on the CCDS that the MAH has submitted for approval. In different regions and countries, some health authorities require the MAH to base their national label on an approved label in a "major market". Often, health authorities only approve variations to the label when there is evidence that the reference market health authority has already approved them. This practice is prevalent in many emerging markets and can be considered a form of labelling harmonisation [4]. Within the EU, participation in specific registration procedures [i.e. mutual recognition procedure (MRP), centralised approval procedure (CAP), decentralised approval procedure (DCP)] across many countries is a further step to harmonisation within an emerging market. However, labelling harmonisation across regions, particularly safety-related information, represents a key factor in promoting patient safety, risk communication, and risk minimisation measures; hence, it is an important topic for consideration in the future.

\subsection{Interchangeability}

Data publishing/exchanging is one of the key things that pharmaceutical companies/health authorities should consider while updating labelling documents. The PSUR worksharing scheme that was adopted within the European Economic Area (EEA), as well as the PSUR synchronisation project, resulted in a reduction of administrative burden [10]. We hope that regulators will also consider the aspect of harmonisation of labels as an essential topic to improve public health.

\section{Conclusion}

The challenges in the field of CCDS maintenance process for generic medicines are complex and an end-to-end solution covering all aspects will be promising. The authors believe that the creation of data exchange platforms creates a widely accepted solution to address challenges in CCDS maintenance and to create high-quality documents across the industry that improves patient health. In addition, authors believe that there is a huge need for a harmonised process for label updates across the industry and clear guidance needs to be provided for pharmaceutical companies in maintaining CCDS updates.

Approaches by MAH might include reduction of administrative and bureaucratic workload by referring to new IT solutions and on other hand support the companies in focusing on medical and scientific evaluation of the available evidence. Although some IT solutions seem to be very promising, the main limiting factor might be budget availability. However, the benefits and risks of public health, litigation 
and reputation of outdated reference safety information far outweigh any financial risks/factors.

The conclusion is that there are many alternatives to the status quo. Not all of the proposed solutions have been or will be successful; some of them may not be applicable, others are over-ambitious or are practically unaffordable for a generic company. However, some methods are pragmatic and have a strong possibility as the operating model of choice. The aim of MAHs should be to work more efficiently to maintain harmonisation, and up-to-date labels for generic substances, to support generic substitution and facilitate choices made by prescribers and patients alike.

Acknowledgements We thank Julie Gabriel for her careful reading of our manuscript and her many insightful comments and suggestions.

\section{Declarations}

Funding This article was not funded.

Conflict of interest The authors work for different departments of a pharmaceutical company undertaking work related to the content of the article.

Ethics approval Not applicable.

Consent to participate Not applicable.

Consent for publication Not applicable.

Data availability Not applicable.

Code availability Not applicable.

Author contributions MM conceived of the presented idea. MM, KKD collected the data and prepared the article. SH and DJL supervised and reviewed this work. All authors discussed the information in the article and contributed to the final manuscript.

Open Access This article is licensed under a Creative Commons Attribution-NonCommercial 4.0 International License, which permits any non-commercial use, sharing, adaptation, distribution and reproduction in any medium or format, as long as you give appropriate credit to the original author(s) and the source, provide a link to the Creative Commons licence, and indicate if changes were made. The images or other third party material in this article are included in the article's Creative
Commons licence, unless indicated otherwise in a credit line to the material. If material is not included in the article's Creative Commons licence and your intended use is not permitted by statutory regulation or exceeds the permitted use, you will need to obtain permission directly from the copyright holder. To view a copy of this licence, visit http://creativecommons.org/licenses/by-nc/4.0/.

\section{References}

1. Agency EM. How to prepare and review a summary of product characteristics. 2019. https://www.ema.europa.eu/en/human-regul atory/marketing-authorisation/product-information/how-preparereview-summary-product-characteristics. Accessed 17 Mar 2020.

2. European Union Pharmacovigilance Legislation. Directive 2001/83/EC of the European Parliament and of the Council. 2001. https://www.ema.europa.eu/en/documents/regulatory-proce dural-guideline/directive-2001/83/ec-european-parliament-counc il-6-november-2001-community-code-relating-medicinal-produ cts-human-use_en.pdf. Accessed 15 Nov 2019.

3. Health Canada. Summary Safety Review-Beta-lactam antibiotics-Assessing the potential risk of severe skin side effects. 2018. https://www.canada.ca/en/health-canada/services/drugs -health-products/medeffect-canada/health-product-infowatch/healt h-product-infowatch-october-2018.html. Accessed 24 Oct 2018.

4. Yoshida S, Matsui R, Kikuchi C. What drives adoption of national labels as global reference labels? A case study with the JPI. Ther Innov Regul Sci. 2018;52(6):724-30. https://doi. org/10.1177/2168479018755084.

5. Dunne SS, Dunne CP. What do people really think of generic medicines? A systematic review and critical appraisal of literature on stakeholder perceptions of generic drugs. BMC Med. 2015;13:173. https://doi.org/10.1186/s12916-015-0415-3.

6. Duke J, Friedlin J, Li X. Consistency in the safety labeling of bioequivalent medications. Pharmacoepidemiol Drug Saf. 2013;22(3):294-301. https://doi.org/10.1002/pds.3351.

7. European Medicines Agency. European Medicines Agency closes PIM project. 2011. https://www.ema.europa.eu/en/news/europeanmedicines-agency-closes-pim-project. Accessed 04 May 2019.

8. Hock S. The company core data sheet in light of XML-authoring and IDMP master data implementation. Ther Innov Regul Sci. 2017;51(3):342-51. https://doi.org/10.1177/2168479016680255.

9. Furlan G. Using resources for scientific-driven pharmacovigilance: from many product safety documents to one product safety master file. Drug Saf. 2012;35(8):615-22. https://doi. org/10.2165/11631290-000000000-00000.

10. European Medicines Agency and Heads of Medicines Agencies. PSUR Work Sharing and Synchronisation Project. 2002. https:// www.hma.eu/348.html. Accessed 15 Nov 2019. 